\title{
Oenological Characteristics of Four Non-Saccharomyces Yeast Strains With $\beta$-Glycosidase Activity
}

\author{
Tao Qin, Jing Liao, Yingyuan Zheng, Wenxia Zhang and Xiuyan Zhang* \\ College of Food Science and Technology, Huazhong Agricultural University, Wuhan, China
}

Non-Saccharomyces yeast with $\beta$-glucosidase activity might positively contribute to the flavor and quality of wines. The contribution of four non-Saccharomyces yeast strains Issatchenkia terricola SLY-4, Pichia kudriavzevii F2-24, P. kudriavzevii F2-16, and Metschnikowia pulcherrima HX-13 with $\beta$-glucosidase activity to the flavor and quality of wine making was studied. Compared with those of $S$. cerevisiae single fermentation, the four non-Saccharomyces yeast strains could grow and consume sugar completely

OPEN ACCESS

Edited by:

Kap-Hoon Han

Woosuk University, South Korea

Reviewed by:

Estéfani García Ríos,

Instituto de Salud Carlos III (ISCIII),

Spain

Hee-Soo Park

Kyungpook National University,

South Korea

${ }^{*}$ Correspondence:

Xiuyan Zhang

xiuyanzhang73@mail.hzau.edu.cn

Specialty section:

This article was submitted to

Food Microbiology,

a section of the journal

Frontiers in Microbiology

Received: 07 November 2020

Accepted: 30 July 2021

Published: 03 September 2021

Citation:

Qin T, Liao J, Zheng Y, Zhang W

and Zhang $X$ (2021) Oenological

Characteristics of Four

Non-Saccharomyces Yeast Strains

With $\beta$-Glycosidase Activity.

Front. Microbiol. 12:626920.

doi: 10.3389/fmicb.2021.626920 with longer fermentation periods, and with no significantly negative effect on chemical characteristics of wines. Moreover, they produced lower content of $\mathrm{C}_{6}$ compounds, benzene derivative, and fatty acid ethyl ester compounds and higher content of terpene, $\beta$-ionone, higher alcohol, and acetate compounds. Different yeast strains produced different aroma compounds profiles. In general, the sensory evaluation score of adding non-Saccharomyces yeast-fermented wine was better than that of $S$. cerevisiae, and I. terricola SLY-4 fermentation received the highest one, followed by P. kudriavzevii F224, P. kudriavzevii F2-16, and M. pulcherrima $\mathrm{HX}-13$ from high to low. The research results provide a theoretical basis for the breeding of non-Saccharomyces yeast and its application in wine making.

Keywords: oenological characteristics, wine, flavor, $\beta$-glucosidase, non-Saccharomyces yeast

\section{INTRODUCTION}

It is an established enological practice to use commercial Saccharomyces cerevisiae to ferment wine. Pure S. cerevisiae fermentation has an easy control fermentation process and a high consistency of product quality between batches, but it is easy to lead poor flavor complexity and varietal aroma characteristics of wine which are mainly contributed by varietal, fermentative, and aging aroma compounds (Pires et al., 2014).

At present, non-Saccharomyces yeast is widely accepted because of its ability to produce aroma compounds and other excellent brewing characteristics, which has been used in pure or mixed fermentation with $S$. cerevisiae to overcome the defect of imperfect wine flavor (Parker et al., 2017; Canonico et al., 2019; Plessis et al., 2019; Binati et al., 2020). The varietal aroma characteristics of wine are mainly contributed by the volatile varietal aroma compounds; however, these compounds often exist as non-volatile glycoside precursors and are odorless. The nonvolatile glycosides can be hydrolyzed by $\beta$-glycosidases and released as volatile compounds 
with flavor (Cabrita et al., 2010; García-Carpintero et al., 2011). $\beta$-Glycosidase from different resources will affect the category and concentration of volatile varietal aroma compounds (Baffi et al., 2011). Generally, single fermentation of S. cerevisiae is weak in liberating these aroma precursors (Boscaino et al., 2019).

Several non-Saccharomyces yeast strains have been confirmed to have $\beta$-glucosidase activity, including Hanseniaspora uvarum, Pichia fermentans, Pichia membranifaciens, Wickerhamomyces anomalus, and Rhodotorula mucilaginosa, and they can improve the content of some volatile aroma compounds such as terpenes and benzene derivatives, imparting fruity and floral flavor profile to wine (Sabel et al., 2014; López et al., 2015, 2016; Ovalle et al., 2016; Ma et al., 2017; Sun et al., 2017). Four nonSaccharomyces yeast strains I. terricola SLY-4, P. kudriavzevii F2-24, P. kudriavzevii F2-16, and M. pulcherrima HX-13 were isolated from vineyards of the Helan Mountain region in Ningxia of China by our research group, which produced 98.51, 76.93, 62.72, and $47.95 \mathrm{U} / \mathrm{l} \beta$-glucosidase activities, respectively (Wang et al., 2018). Adding crude extraction of $\beta$-glucosidases from I. terricola SLY-4, P. kudriavzevii F2-24, and M. pulcherrima HX-13 into must could increase the content of terpenes, esters, and fatty acids and enhance the fruity and floral aroma of wine fermented by $S$. cerevisiae. Wines using different crude $\beta$-glucosidase extractions presented distinct volatile compound profiles and varied typical flavor characteristics (Zhang et al., 2020). These results indicated that $\beta$-glucosidases from the four non-Saccharomyces yeast strains could enhance the content of aroma compounds with different profiles and improve the fruity and floral aroma of wine. However, the positive or negative effects of these four non-Saccharomyces yeast strains as main brewing yeast on wine-making are not yet known.

To investigate the effects of pure fermentations of $I$. terricola SLY-4, P. kudriavzevii F2-24, P. kudriavzevii F2-16, or M. pulcherrima HX-13 on flavor complexity and varietal aroma characteristics of wines, the process and the quality of their pure fermentations will be analyzed. Research results will provide some references of using non-Saccharomyces yeast strains to improve the flavor complexity and varietal characteristics of wines.

\section{MATERIALS AND METHODS}

\section{Strains and Medium}

I. terricola SLY-4, P. kudriavzevii F2-24, P. kudriavzevii F2-16, and $M$. pulcherrima $\mathrm{HX}-13$ were isolated from vineyards of the Helan Mountain region in Ningxia of China. They have been identified through sequence analysis of the 26S rDNA D1/D2 domain and kept in our lab. Reference strains were purchased from the China General Microbiological Culture Collection Center (CGMCC 2.3216 Issatchenkia terricola; CGMCC 2.454 Pichia kudriavzevii; CGMCC 2.3776 Metschnikowia pulcherrima). Saccharomyces cerevisiae was a commercial strain Actiflore $^{\circledR}$ F33 (Laffort, France).

Yeast extract peptone dextrose medium (YPD, $10 \mathrm{~g} / \mathrm{l}$ yeast extract, $20 \mathrm{~g} / \mathrm{l}$ peptone, $20 \mathrm{~g} / \mathrm{l}$ glucose, and $20 \mathrm{~g} / \mathrm{l}$ agar) was used to inoculate preparation and yeast cell count.

\section{Laboratory-Scale Fermentation of Wines}

Cabernet Sauvignon grapes from a vineyard of the Helan Mountain region in Ningxia of China were destemmed and crushed into must (239.9 g/l total sugar calculated as glucose and $7.1 \mathrm{~g} / \mathrm{l}$ total acid calculated as tartaric acid, $\mathrm{pH}$ 3.96). Eight hundred milliliters of must was filled into a 1.0-1 glass bottle, pasteurized at $68.5^{\circ} \mathrm{C}$ for $30 \mathrm{~min}$. After adding $50 \mathrm{mg} / \mathrm{l}$ $\mathrm{SO}_{2}$, the must was macerated at $4^{\circ} \mathrm{C}$ for $12 \mathrm{~h}$, and yeast cells were inoculated at $10^{6} \mathrm{CFU} / \mathrm{ml}$ and fermented at $20^{\circ} \mathrm{C}$ without agitation. Each kind of yeast was inoculated in triplicate.

\section{Viable Yeast Cell Counting}

Samples were taken during fermentation every day and concurrently diluted onto a YPD plate, then incubated at $28^{\circ} \mathrm{C}$ for 3 days (Suárez et al., 2007). Colonies on the YPD plate were counted as the viable cells of S. cerevisiae or non-Saccharomyces yeast. Each sample was analyzed in triplicate from the bottles.

\section{Analytical Determination of Wines}

The contents of residual sugar, alcohol, total acid, and volatile acid of wine were analyzed through methods recommended by the International Organization of the Vine and Wine (OIVMA-AS311-02: R2009, 2009; OIV-MA-AS313-01: R2015, 2015; OIV-MA-AS313-02: R2015, 2015; OIV-MA-AS312-01A: R2016, 2016). The residual sugar contents were expressed as glucose $(\mathrm{g} / \mathrm{L})$. The total acid content was expressed as tartaric acid $(\mathrm{g} / \mathrm{L})$, and the volatile acid content was expressed as acetic acid $(\mathrm{g} / \mathrm{L})$. Each wine sample was measured in triplicates from the bottles.

The extraction of volatile aroma compounds from wine was conducted by headspace solid-phase microextraction with 50/30 $\mu \mathrm{m}$ divinylbenzene carboxen polydimethylsiloxane (DVB/CAR/PDMS) fiber (Supelco, Bellefonte, PA, United States). The extracted volatile compounds were analyzed on an Agilent $6890 \mathrm{~N}$ gas chromatograph (GC) coupled to an Agilent 5975B mass spectrometer with a DB-5 capillary column (30 $\mathrm{m} \times 0.32 \mathrm{~mm} \times 0.25 \mu \mathrm{m})$. An 8-ml sample containing $0.45 \mathrm{~g}$ cyclohexanone (internal standard) and $2 \mathrm{~g} \mathrm{NaCl}$ was put in a 20-ml headspace bottle and stirred by a magnetic bar at $40^{\circ} \mathrm{C}$ for $15 \mathrm{~min}$. After that, the fiber was exposed to the headspace of bottle for $30 \mathrm{~min}$ and immediately desorbed in an injector at $250^{\circ} \mathrm{C}$ for $3 \mathrm{~min}$. The operating conditions of $\mathrm{GC}$ were the following: initial temperature $40^{\circ} \mathrm{C}$, increased to $130^{\circ} \mathrm{C}$ at $3^{\circ} \mathrm{C} / \mathrm{min}$, then to $250^{\circ} \mathrm{C}$ at $4^{\circ} \mathrm{C} / \mathrm{min}$. The injector and detector were set at 250 and $260^{\circ} \mathrm{C}$, respectively. The mass spectrometry was operated in electron impact ionization mode at $70 \mathrm{eV}$, and ion source temperature was $250^{\circ} \mathrm{C}$. Detection was carried out in full-scan mode over a range of 30-350 u/s. Compounds were identified by comparing their retention time with MS fragmentation patterns which were obtained from databases Wiley 7.0 and NIST05. All volatile compounds were semi-quantified through the following formula:

$$
\text { Compound content }(m g / m L)=
$$

GC peak area of compound $\times$ Quantity of cyclohexanone $(\mathrm{mg})$ GC peak area of cyclohexanone $\times$ volume of sample $(m L)$ 


\section{Sensory Evaluation of Wines}

The sensory evaluation was performed as described by Belda et al. (2015) with modification. Twenty milliliters of wine was poured into wine glasses and presented in random order. The preferences for appearance, aroma (fruity, floral, and green), and taste of the wine were scored from 0 (weak) to 9 (intense) by a well-trained panelist (six females and four males) from Huazhong Agricultural University, respectively. The final score of each sensory characteristic was the mean value of 10 scores given by 10 assessors, respectively.

\section{Data Statistics Processing and Analysis}

Data and chart were performed by Microsoft Office 2010 and GraphPad Prism 6.0. One-way ANOVA was completed by SPSS 19.0 software (SPSS Inc., Chicago, IL, United States). Principal component analysis (PCA) was performed by SIMCA-P 14.1 (Umetrics AB, Umea, Sweden).

\section{RESULTS}

\section{Growth and Sugar Consumption Kinetics of Yeast Strains During Wine Fermentation}

The growth and sugar consumption kinetics of yeast strains indicated that four non-Saccharomyces yeast strains could grow normally during wine fermentation (Figure 1). Compared with that of S. cerevisiae $\left(2.25 \times 10^{9} \mathrm{cells} / \mathrm{ml}\right)$, the biomasses of four non-Saccharomyces yeast strains were higher. M. pulcherrima HX-13 had the highest biomass $\left(11.45 \times 10^{9}\right.$ cells $\left./ \mathrm{ml}\right)$, followed by $I$. terricola SLY-4 $\left(4.8 \times 10^{9}\right.$ cells $\left./ \mathrm{ml}\right), P$. kudriavzevii F2$16\left(3.05 \times 10^{9} \mathrm{cells} / \mathrm{ml}\right)$, and P. kudriavzevii F2-24 $\left(2.8 \times 10^{9}\right.$ cells $/ \mathrm{ml}$ ). Compared with that of $S$. cerevisiae (7 days), the fermentation periods of the four non-Saccharomyces yeasts were longer (9-13 days). M. pulcherrima HX-13 had the shortest fermentation period (9 days) among the non-Saccharomyces yeasts, followed by I. terricola SLY-4 (10 days), P. kudriavzevii F2-24 (10 days), and P. kudriavzevii F2-16 (13 days).

\section{Chemical Characteristics of Wines Fermented by Yeast Strains With $\beta$-Glucosidase Activity}

The chemical characteristics of wines fermented by different yeast strains showed that all the fermentations contained 2.71-3.64 g/l residue sugar (expressed as glucose), about $12 \%$ ethanol (v/v), 5.13-5.44 g/l total acid (expressed as tartaric acid), and 0.23$0.31 \mathrm{~g} / \mathrm{l}$ volatile acid (expressed as acetic acid) (Table 1). These results indicated there was no negative effect on the chemical characteristics of wines.

\section{Volatile Compounds of Wines Fermented by Yeast Strains With $\beta$-Glucosidase Activity}

The total ion current chromatograms of gas chromatographymass spectrometry (GC-MS) analysis for all the fermentations indicated that different fermentations had unique chromatogram profiles. Fifty-three kinds of volatile compounds were classified into variety aroma compounds and fermentative aroma compounds. Eleven variety aroma components were clustered into $\mathrm{C}_{6}$ compound, terpene, norisoprenoid, and benzene derivative compound. Forty-two fermentative aroma components were clustered into compounds of higher alcohol, fatty acid, ester (acetic ester, fatty acid ethyl ester, and other ester), aldehyde, and ketone (Table 2).

The effects of non-Saccharomyces yeast strains on the aroma compounds were evaluated as follows.

Compared with those of the S. cerevisiae single fermentation, the total contents of varietal aroma compounds in non-Saccharomyces yeast fermentations were lower (45.49$62.64 \mathrm{mg} / \mathrm{l})$. Among the varietal aroma compounds, lower contents of $\mathrm{C}_{6}$ compounds $(0.21-0.54 \mathrm{mg} / \mathrm{l})$ and benzene derivative compounds (44.68-61.49 $\mathrm{mg} / \mathrm{l})$ and higher contents of terpene $(0.26-0.89 \mathrm{mg} / \mathrm{l})$ and $\mathrm{C}_{13}$-norisoprenoid compounds (0.01-0.03 mg/l) were produced. Limonene, linalool, citronellol, nerol, $\beta$-ionone, phenylethyl alcohol, and phenylethyl acetate were the main odor active variety aroma compounds $(\mathrm{OAV}>1)$ (Table 2 and Figure 2).

Compared with the $S$. cerevisiae single fermentation, the four non-Saccharomyces yeast fermentations contained higher contents of fermentative aroma compounds (499.00$636.72 \mathrm{mg} / \mathrm{l}$ ) (Table 2 and Figure 2), especially higher alcohol (233.21-308.67 mg/l), and ester compounds (250.04$319.98 \mathrm{mg} / \mathrm{l})$, and they produced higher concentrations of acetate compounds (195.87-242.58 mg/l) and lower concentrations of fatty acid ethyl ester compounds (32.11-77.40 mg/l). Isoamyl alcohol, isobutanol, 2-methylpentanol, 1-octanal, ethyl acetate, isobutyl acetate, isoamyl acetate, 2-methylbutyl acetate, ethyl butyrate, ethyl hexanoate, ethyl octanoate, ethyl 9-decenoate, ethyl decanoate, ethyl laurate, isoamyl caprylate, and isoamyl caproate were the main odor active fermentation aroma compounds.

\section{PCA of Volatile Compounds From Wines Fermented by Yeast Strains With $\beta$-Glucosidase Activity}

A principal component analysis (PCA) was carried out to reveal the correlation and segregation of volatile compounds with different yeast strain fermentations. Here $68.2 \%$ of variance was explained, and PC1 and PC2 accounted for $41 \%$ and $27.2 \%$ of variance, respectively. The $P$. kudriavzevii F2-16 fermentation and the P. kudriavzevii F2-24 fermentation were mainly grouped with varietal aroma compounds such as limonene, linalool, citronellol, and some kinds of fermentative aroma compounds such as 1-octanol, 2-methyl-1-butanol, isopentanoic acid, and 2methylbutyric acid. The I. terricola SLY-4 fermentation and the M. pulcherrima HX-13 fermentation were closely clustered with various fermentative aroma compounds such as 2,3-butanediol, isoamyl alcohol, isobutyl acetate, acetic acid 2-methyl, ethyl butyrate, octanoic acid, 1-decanol, ethyl decanoate, phenylethyl acetate, and hexanoic acid. S. cerevisiae fermentation was grouped with some fermentative aroma compounds such as 


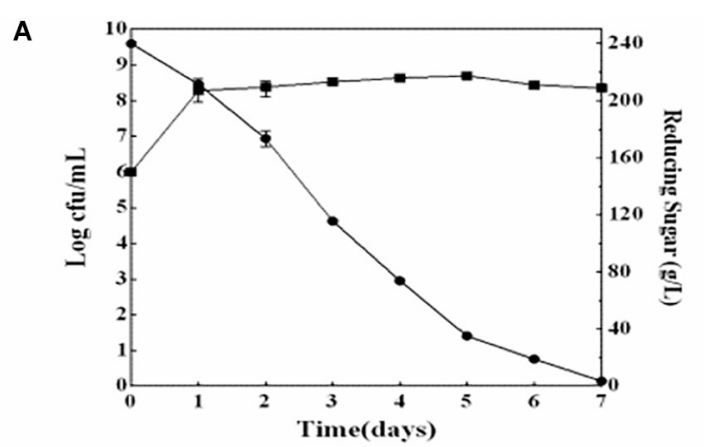

B

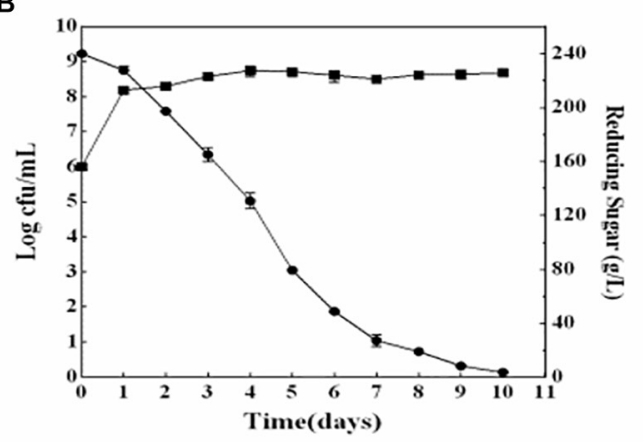

D

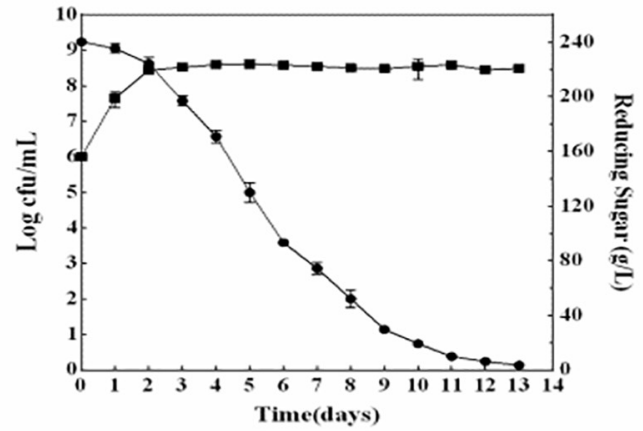

C

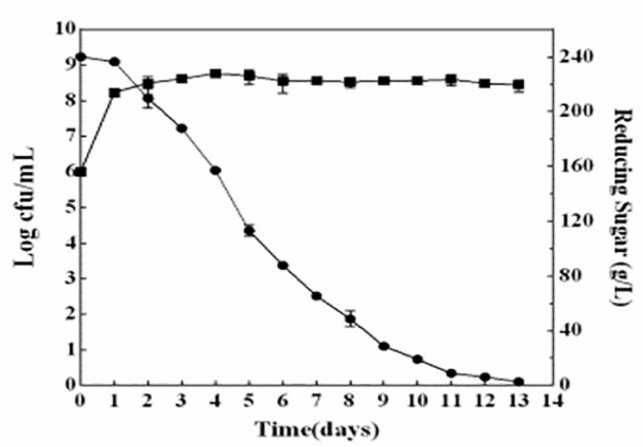

E

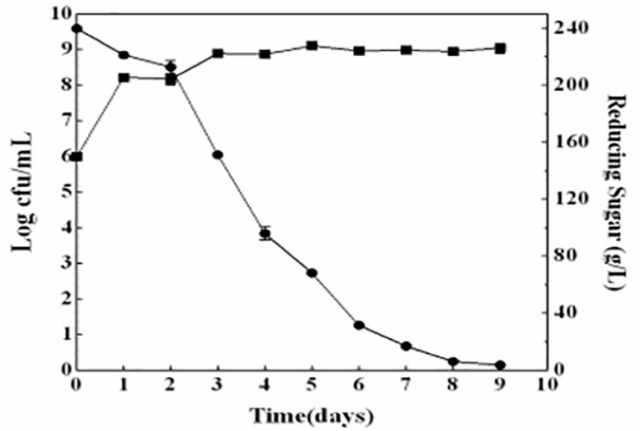

FIGURE 1 | Growth and sugar consumption kinetics of yeast strains during wine fermentation. (A) S. cerevisiae fermentation; (B) I. terricola SLY-4 fermentation; (C) P. kudriavzevii F2-24 fermentation; (D) P. kudriavzevii F2-16 fermentation; (E) M. pulcherrima HX-13 fermentation. - - -Growth kinetics of yeast strains - - -Sugar consumption kinetics of yeast strains.

TABLE 1 | Chemical characteristics of wines fermented by yeast with $\beta$-glycosidase activity.

\begin{tabular}{|c|c|c|c|c|c|}
\hline Wines & Time (days) & Residual sugar (g/L) & Alcohol content $(\%, v / v)$ & Total acid (g/L) & Volatile acid (g/L) \\
\hline SCw & 7 & $3.57 \pm 0.19^{a}$ & $12.85 \pm 0.36^{a}$ & $5.44 \pm 0.19^{a}$ & $0.23 \pm 0.01^{b}$ \\
\hline SLY-4w & 10 & $3.64 \pm 0.07^{a}$ & $12.91 \pm 0.28^{a}$ & $5.44 \pm 0.19^{a}$ & $0.31 \pm 0.01^{a}$ \\
\hline F2-16w & 13 & $3.53 \pm 0.20^{\mathrm{a}}$ & $12.08 \pm 0.38^{a}$ & $5.13 \pm 0.18^{b}$ & $0.25 \pm 0.01^{b}$ \\
\hline$H X-13 w$ & 9 & $3.50 \pm 0.30^{a}$ & $12.96 \pm 0.13^{\mathrm{a}}$ & $5.31 \pm 0.11^{b}$ & $0.24 \pm 0.00^{b}$ \\
\hline
\end{tabular}

Different inline letters in the same row of values indicate significant differences determined by Duncan test at $95 \%$ confidence level.

SCW, S. cerevisiae fermentation; SLY-4w, I. terricola SLY-4 fermentation; F2-24w, P. kudriavzevii F2-24 fermentation; F2-16w, P. kudriavzevii F2-16 fermentation; HX-13w, M. pulcherrima $H X-13$ fermentation. 
TABLE 2 | Volatile compounds in different wines ( $\mathrm{mg} / \mathrm{L}$ ).

\begin{tabular}{|c|c|c|c|c|c|c|c|c|}
\hline \multirow[t]{2}{*}{ Compounds } & \multicolumn{5}{|c|}{ Concentration (mg/L) } & \multirow[t]{2}{*}{ Odor threshold } & \multirow[t]{2}{*}{ OAV } & \multirow[t]{2}{*}{ Odors descriptions } \\
\hline & $\mathrm{scw}$ & SLY-4w & F2-24w & F2-16w & $H X-13 w$ & & & \\
\hline Varietal aroma & $85.29 \pm 12.88^{a}$ & $60.16 \pm 0.40^{\mathrm{b}}$ & $45.49 \pm 6.18^{c}$ & $62.64 \pm 0.53^{b}$ & $57.89 \pm 4.23^{b}$ & & & \\
\hline $\mathrm{C}_{6}$ compounds & $0.78 \pm 0.04^{a}$ & $0.54 \pm 0.02^{b}$ & $0.21 \pm 0.04^{d}$ & $0.25 \pm 0.01^{d}$ & $0.33 \pm 0.00^{c}$ & & & \\
\hline 1-Hexanol & $0.36 \pm 0.05^{a}$ & $0.02 \pm 0.01^{\mathrm{C}}$ & $0.03 \pm 0.01^{c}$ & $0.06 \pm 0.02^{C}$ & $0.11 \pm 0.01^{\mathrm{b}}$ & 8 & $<0.1$ & Green, herb \\
\hline cis-2-Hexen-1-ol & $0.42 \pm 0.02^{c}$ & $0.52 \pm 0.03^{b}$ & $0.19 \pm 0.05^{d}$ & $0.20 \pm 0.01^{d}$ & $0.23 \pm 0.01^{d}$ & 0.4 & $>0.1$ & Green, herb \\
\hline Terpenes & $0.04 \pm 0.01^{e}$ & $0.26 \pm 0.01^{d}$ & $0.59 \pm 0.01^{b}$ & $0.89 \pm 0.01^{a}$ & $0.33 \pm 0.04^{c}$ & & & \\
\hline Limonene & $0.00 \pm 0.00^{c}$ & $0.00 \pm 0.00^{c}$ & $0.15 \pm 0.01^{b}$ & $0.22 \pm 0.01^{a}$ & $0.00 \pm 0.00^{\circ}$ & 0.1 & $>1$ & Lemon, citrus \\
\hline Linalool & $0.01 \pm 0.00^{e}$ & $0.10 \pm 0.01^{c}$ & $0.17 \pm 0.01^{\mathrm{a}}$ & $0.12 \pm 0.01^{b}$ & $0.02 \pm 0.01^{d}$ & 0.025 & $>1$ & Muscat, flowery, fruity \\
\hline Citronellol & $0.02 \pm 0.01^{d}$ & $0.09 \pm 0.01^{c}$ & $0.17 \pm 0.01^{b}$ & $0.42 \pm 0.01^{a}$ & $0.17 \pm 0.01^{\mathrm{b}}$ & 0.01 & $>1$ & Citrus \\
\hline Nerol & $0.01 \pm 0.00^{d}$ & $0.08 \pm 0.02^{\mathrm{C}}$ & $0.11 \pm 0.01^{b}$ & $0.14 \pm 0.02^{a}$ & $0.14 \pm 0.03^{a}$ & 0.03 & $>1$ & Citrus \\
\hline $\mathrm{C}_{13}$-Norisoprenoids & $0.00 \pm 0.00^{c}$ & $0.03 \pm 0.01^{a}$ & $0.01 \pm 0.00^{b}$ & $0.02 \pm 0.01^{b}$ & $0.00 \pm 0.00^{c}$ & & & \\
\hline$\beta$-lonone & $0.00 \pm 0.00^{c}$ & $0.03 \pm 0.01^{a}$ & $0.01 \pm 0.00^{b}$ & $0.02 \pm 0.01^{b}$ & $0.00 \pm 0.00^{c}$ & $9^{*} 10^{-5}$ & $>1$ & Raspberry, violet, sweet \\
\hline Benzene derivatives & $84.51 \pm 12.91^{a}$ & $59.34 \pm 0.41^{b}$ & $44.68 \pm 6.21^{c}$ & $61.49 \pm 0.56^{b}$ & $57.23 \pm 4.27^{\mathrm{b}}$ & & & fruity \\
\hline Benzaldehyde & $0.29 \pm 0.06^{b}$ & $0.81 \pm 0.02^{\mathrm{a}}$ & $0.00 \pm 0.00^{c}$ & $0.00 \pm 0.00^{C}$ & $0.87 \pm 0.06^{a}$ & 2 & $<0.1$ & \\
\hline Benzyl alcohol & $0.00 \pm 0.00^{b}$ & $0.30 \pm 0.01^{\mathrm{a}}$ & $0.00 \pm 0.00^{b}$ & $0.00 \pm 0.00^{b}$ & $0.00 \pm 0.00^{\mathrm{b}}$ & 200 & $<0.1$ & Almond, fatty \\
\hline Phenethyl alcohol & $72.16 \pm 11.14^{a}$ & $30.99 \pm 1.56^{d}$ & $40.35 \pm 6.09^{c}$ & $55.14 \pm 0.75^{b}$ & $33.24 \pm 4.92^{d}$ & 14 & $>1$ & Rose, soft tommy \\
\hline Phenylethyl acetate & $12.07 \pm 1.71^{\mathrm{C}}$ & $27.25 \pm 2.00^{\mathrm{a}}$ & $4.33 \pm 0.12^{d}$ & $6.35 \pm 0.20^{d}$ & $23.13 \pm 0.59^{\mathrm{b}}$ & 0.25 & $>1$ & Floral, rose \\
\hline Fermentative aroma & $404.89 \pm 1.45^{d}$ & $499.00 \pm 9.53^{c}$ & $530.83 \pm 14.26^{b}$ & $524.77 \pm 23.19^{b}$ & $636.72 \pm 12.92^{a}$ & & & \\
\hline Higher alcohols & $202.03 \pm 1.93^{\mathrm{e}}$ & $233.21 \pm 1.86^{d}$ & $273.88 \pm 7.06^{c}$ & $285.47 \pm 4.60^{b}$ & $308.67 \pm 10.16^{a}$ & & & \\
\hline 1-Propanol & $2.51 \pm 0.17^{c}$ & $6.89 \pm 0.02^{b}$ & $2.85 \pm 0.49^{c}$ & $8.30 \pm 0.41^{a}$ & $0.00 \pm 0.00^{d}$ & 306 & $<0.1$ & Fresh, alcohol \\
\hline Isobutyl alcohol & $8.06 \pm 0.61^{d}$ & $10.73 \pm 0.52^{d}$ & $29.50 \pm 1.06^{c}$ & $61.01 \pm 4.47^{b}$ & $73.57 \pm 3.23^{\mathrm{a}}$ & 40 & & Mild sweet, alcohol \\
\hline 1-Butanol & $0.41 \pm 0.00^{c}$ & $0.85 \pm 0.17^{\mathrm{ab}}$ & $0.69 \pm 0.13^{b}$ & $1.00 \pm 0.15^{a}$ & $0.00 \pm 0.00^{d}$ & 150 & $<0.1$ & $\begin{array}{l}\text { Medicinal, fusel, } \\
\text { pungency }\end{array}$ \\
\hline Isoamyl alcohol & $120.46 \pm 3.21^{\mathrm{b}}$ & $167.28 \pm 0.05^{a}$ & $172.69 \pm 7.57^{a}$ & $109.57 \pm 3.90^{c}$ & $169.06 \pm 1.60^{\mathrm{a}}$ & 30 & $>1$ & $\begin{array}{l}\text { Alcohol, harsh, bitter, } \\
\text { banana }\end{array}$ \\
\hline 2-Methyl-1-butanol & $68.74 \pm 0.50^{b}$ & $45.36 \pm 1.20^{\circ}$ & $66.97 \pm 0.00^{b}$ & $102.68 \pm 3.11^{\mathrm{a}}$ & $64.47 \pm 14.99^{b}$ & 65 & $>0.1$ & \\
\hline 2,3-Butanediol & $0.77 \pm 0.04^{c}$ & $1.26 \pm 0.01^{b}$ & $0.64 \pm 0.19^{c}$ & $1.67 \pm 0.21^{a}$ & $1.44 \pm 0.02^{b}$ & 120 & $<0.1$ & $\begin{array}{l}\text { Butter, creamy, } \\
\text { chemical }\end{array}$ \\
\hline 3-Methyl-1-pentanol & $0.56 \pm 0.10^{\mathrm{a}}$ & $0.36 \pm 0.01^{b}$ & $0.00 \pm 0.00^{c}$ & $0.00 \pm 0.00^{C}$ & $0.00 \pm 0.00^{c}$ & 0.5 & $>1$ & Fusel \\
\hline 1-Octanol & $0.27 \pm 0.03^{c}$ & $0.50 \pm 0.05^{b}$ & $0.44 \pm 0.01^{b}$ & $1.26 \pm 0.15^{a}$ & $0.14 \pm 0.03^{d}$ & 0.9 & $0.1-1$ & Waxy \\
\hline 1-Decanol & $0.27 \pm 0.08^{a}$ & $0.00 \pm 0.00^{c}$ & $0.12 \pm 0.01^{d}$ & $0.00 \pm 0.00^{\circ}$ & $0.00 \pm 0.00^{c}$ & 0.4 & $0.1-1$ & Citrus, fatty \\
\hline Fatty acids & $5.11 \pm 0.06^{c}$ & $9.16 \pm 0.33^{\mathrm{a}}$ & $4.44 \pm 0.14^{d}$ & $5.91 \pm 0.32^{b}$ & $5.28 \pm 0.22^{c}$ & & & \\
\hline Isobutyric acid & $0.18 \pm 0.01^{b}$ & $0.00 \pm 0.00^{c}$ & $0.00 \pm 0.00^{c}$ & $0.68 \pm 0.03^{a}$ & $0.00 \pm 0.00^{c}$ & 8.1 & $<0.1$ & Phenol, chemical, fatty \\
\hline 2-Methylbutyric acid & $0.32 \pm 0.04^{b}$ & $0.00 \pm 0.00^{c}$ & $0.29 \pm 0.00^{b}$ & $1.26 \pm 0.11^{\mathrm{a}}$ & $0.00 \pm 0.00^{\circ}$ & 0.25 & $>1$ & Cheese \\
\hline Isopentanoic acid & $0.00 \pm 0.00^{c}$ & $0.63 \pm 0.07^{b}$ & $0.47 \pm 0.02^{b}$ & $1.02 \pm 0.49^{a}$ & $0.00 \pm 0.00^{c}$ & 0.033 & $>1$ & Sweaty feet \\
\hline Hexanoic acid & $1.54 \pm 0.06^{b}$ & $2.21 \pm 0.03^{a}$ & $1.52 \pm 0.06^{b}$ & $1.13 \pm 0.00^{d}$ & $2.25 \pm 0.10^{\mathrm{a}}$ & 0.42 & $>1$ & Cheese, rancid \\
\hline Octanoic acid & $3.01 \pm 0.04^{c}$ & $5.84 \pm 0.20^{a}$ & $2.06 \pm 0.05^{e}$ & $1.83 \pm 0.04^{f}$ & $2.57 \pm 0.11^{d}$ & 0.5 & $>1$ & $\begin{array}{l}\text { Rancid, harsh, cheese, } \\
\text { fatty acid }\end{array}$ \\
\hline Decanoic acid & $0.06 \pm 0.00^{\mathrm{cd}}$ & $0.48 \pm 0.03^{b}$ & $0.11 \pm 0.01^{c}$ & $0.00 \pm 0.00^{d}$ & $0.46 \pm 0.01^{\mathrm{b}}$ & 1 & $<0.1$ & Unpleasant \\
\hline Esters & $194.88 \pm 3.24^{\mathrm{e}}$ & $251.99 \pm 10.90^{b}$ & $250.04 \pm 21.92^{b}$ & $231.50 \pm 19.11^{\mathrm{b}}$ & $319.98 \pm 23.37^{a}$ & & & \\
\hline Acetic esters & $90.82 \pm 3.40^{c}$ & $203.12 \pm 4.25^{b}$ & $217.94 \pm 25.24^{\mathrm{ab}}$ & $195.87 \pm 14.59^{b}$ & $242.58 \pm 17.28^{a}$ & & & \\
\hline
\end{tabular}

$0.06 \pm 0.02^{\circ}$

$0.20 \pm 0.01$

$0.22 \pm 0.01^{a}$

$0.02 \pm 0.01^{b}$

$0.00 \pm 0.00^{\circ}$

$1.00 \pm 0.15^{a}$

$2.68+3.11^{a}$

$.26 \pm 0.15$

$1.02 \pm 0.49^{a}$

$1.13 \pm 0.00^{\circ}$

$195.87 \pm 14.59$ 
TABLE 2 | Continued

\begin{tabular}{|c|c|c|c|c|c|c|c|c|}
\hline \multirow[t]{2}{*}{ Compounds } & \multicolumn{5}{|c|}{ Concentration (mg/L) } & \multirow[t]{2}{*}{ Odor threshold } & \multirow[t]{2}{*}{ OAV } & \multirow{2}{*}{$\begin{array}{l}\text { Odors } \\
\text { descriptions }\end{array}$} \\
\hline & scw & SLY-4w & F2-24w & F2-16w & $H X-13 w$ & & & \\
\hline Ethyl acetate & $50.46 \pm 2.14^{d}$ & $107.46 \pm 8.00^{\mathrm{bc}}$ & $143.02 \pm 10.44^{a}$ & $112.46 \pm 11.00^{b}$ & $94.47 \pm 11.00^{c}$ & 7.5 & $>1$ & Fruity, sweet \\
\hline Propyl acetate & $0.00 \pm 0.00^{f}$ & $0.41 \pm 0.00^{c}$ & $0.33 \pm 0.01^{d}$ & $1.13 \pm 0.00^{a}$ & $0.70 \pm 0.02^{b}$ & 4.7 & $<1$ & Fruity \\
\hline Isobutyl acetate & $0.63 \pm 0.07^{d}$ & $1.27 \pm 0.15^{c}$ & $0.82 \pm 0.05^{d}$ & $2.53 \pm 0.40^{b}$ & $10.23 \pm 0.26^{a}$ & 1.6 & $>0.1$ & $\begin{array}{l}\text { Garnetberry, fruity, } \\
\text { flowery }\end{array}$ \\
\hline Isoamyl acetate & $33.70 \pm 0.94^{d}$ & $85.91 \pm 5.35^{b c}$ & $71.47 \pm 14.98^{\mathrm{b}}$ & $75.31 \pm 3.15^{c}$ & $117.79 \pm 6.00^{\mathrm{a}}$ & 0.03 & $>1$ & Banana \\
\hline 2-Methylbutyl acetate & $5.78 \pm 2.11^{\mathrm{bc}}$ & $7.53 \pm 1.32^{\mathrm{b}}$ & $2.30 \pm 0.12^{d}$ & $4.45 \pm 0.05^{\mathrm{c}}$ & $19.39 \pm 0.00^{\mathrm{a}}$ & $0.02-0.05$ & $>1$ & Fruity \\
\hline Pentanol acetate & $0.00 \pm 0.00^{b}$ & $0.18 \pm 0.05^{a}$ & $0.00 \pm 0.00^{b}$ & $0.00 \pm 0.00^{\mathrm{b}}$ & $0.00 \pm 0.00^{b}$ & & & \\
\hline 3-Methylpentyl acetate & $0.00 \pm 0.00^{b}$ & $0.37 \pm 0.07^{a}$ & $0.00 \pm 0.00^{b}$ & $0.00 \pm 0.00^{\mathrm{b}}$ & $0.00 \pm 0.00^{b}$ & & & \\
\hline Hexyl acetate & $0.19 \pm 0.03^{b}$ & $0.00 \pm 0.00^{\circ}$ & $0.00 \pm 0.00^{c}$ & $0.00 \pm 0.00^{c}$ & $0.00 \pm 0.00^{\circ}$ & 1.5 & $0.1-1$ & Pleasant fruity, pear \\
\hline Octyl acetate & $0.06 \pm 0.00^{b}$ & $0.00 \pm 0.00^{\circ}$ & $0.00 \pm 0.00^{c}$ & $0.00 \pm 0.00^{c}$ & $0.00 \pm 0.00^{\circ}$ & & & \\
\hline Fatty acid ethyl esters & $101.42 \pm 0.20^{\mathrm{a}}$ & $48.87 \pm 6.65^{c}$ & $32.11 \pm 3.32^{d}$ & $35.63 \pm 4.52^{\mathrm{d}}$ & $77.40 \pm 6.09^{b}$ & & & \\
\hline Ethyl propanoate & $0.25 \pm 0.02^{\mathrm{d}}$ & $0.00 \pm 0.00^{e}$ & $0.48 \pm 0.02^{c}$ & $1.48 \pm 0.25^{a}$ & $1.10 \pm 0.06^{b}$ & $1.8-2.1$ & $0.1-1$ & \\
\hline Ethyl butyrate & $2.35 \pm 0.10^{b}$ & $1.81 \pm 0.54^{b c}$ & $1.45 \pm 0.39^{c}$ & $2.10 \pm 0.09^{b}$ & $5.47 \pm 0.50^{a}$ & 0.02 & $>1$ & $\begin{array}{l}\text { Sour fruit, } \\
\text { strawberry, fruity }\end{array}$ \\
\hline Ethyl hexanoate & $44.99 \pm 0.34^{a}$ & $12.43 \pm 2.10^{d}$ & $11.24 \pm 1.09^{d}$ & $10.67 \pm 1.22^{d}$ & $31.77 \pm 2.32^{b}$ & 0.014 & $>1$ & $\begin{array}{l}\text { Green apple, fruity, } \\
\text { strawberry, anise }\end{array}$ \\
\hline Ethyl heptanoate & $38.78 \pm 0.54^{a}$ & $16.47 \pm 1.12^{\mathrm{C}}$ & $11.58 \pm 1.02^{d}$ & $11.95 \pm 0.70^{d}$ & $23.47 \pm 2.42^{b}$ & 0.005 & $>1$ & $\begin{array}{l}\text { Fruity, sweet, anise, } \\
\text { wax }\end{array}$ \\
\hline Ethyl octanoate & $0.06 \pm 0.01^{b}$ & $0.00 \pm 0.00^{\circ}$ & $0.00 \pm 0.00^{c}$ & $0.00 \pm 0.00^{C}$ & $0.00 \pm 0.00^{c}$ & 1.3 & $<0.1$ & banana \\
\hline Ethyl non-anoate & $1.19 \pm 0.06^{\mathrm{a}}$ & $0.19 \pm 0.03^{c}$ & $0.00 \pm 0.00^{c}$ & $0.00 \pm 0.00^{c}$ & $0.43 \pm 0.02^{b}$ & 0.1 & $>1$ & Green, fruity, fatty \\
\hline Ethyl 9-decenoate & $8.09 \pm 0.26^{c}$ & $12.39 \pm 1.71^{\mathrm{b}}$ & $4.67 \pm 0.55^{d}$ & $5.06 \pm 1.91^{d}$ & $9.65 \pm 0.49^{c}$ & 0.1 & $>1$ & $\begin{array}{l}\text { Fruity, fatty, } \\
\text { pleasant, wax flavor }\end{array}$ \\
\hline Ethyl decanoate & $4.04 \pm 0.62^{b}$ & $3.48 \pm 0.83^{b}$ & $1.73 \pm 0.14^{c}$ & $2.16 \pm 0.15^{c}$ & $3.26 \pm 0.29^{b}$ & 1.5 & $>1$ & Waxy \\
\hline Ethyl dodecanoate & $0.64 \pm 0.05^{b}$ & $0.59 \pm 0.03^{c}$ & $0.18 \pm 0.01^{\mathrm{e}}$ & $0.75 \pm 0.01^{\mathrm{a}}$ & $0.30 \pm 0.02^{d}$ & 2 & $0.1-1$ & Fatty, butter \\
\hline Ethyl myristate & $0.76 \pm 0.03^{b}$ & $0.63 \pm 0.18^{b}$ & $0.36 \pm 0.09^{c}$ & $0.34 \pm 0.08^{c}$ & $1.01 \pm 0.01^{\mathrm{a}}$ & $100-200$ & $<0.1$ & Green, fruity \\
\hline Diethyl succinate & $0.30 \pm 0.01^{d}$ & $0.90 \pm 0.12^{\mathrm{b}}$ & $0.44 \pm 0.03^{c}$ & $1.14 \pm 0.13^{a}$ & $0.98 \pm 0.01^{\mathrm{b}}$ & 1.5 & $0.1-1$ & Fatty \\
\hline Ethyl palmitate & $38.78 \pm 0.54^{a}$ & $16.47 \pm 1.12^{\mathrm{C}}$ & $11.58 \pm 1.02^{d}$ & $11.95 \pm 0.70^{d}$ & $23.47 \pm 2.42^{b}$ & 0.005 & $>1$ & $\begin{array}{l}\text { Fruity, sweet, anise, } \\
\text { wax }\end{array}$ \\
\hline Other esters & $2.64 \pm 0.04^{\mathrm{a}}$ & $0.00 \pm 0.00^{c}$ & $0.00 \pm 0.00^{c}$ & $0.00 \pm 0.00^{c}$ & $0.00 \pm 0.00^{c}$ & & & \\
\hline Isoamyl octanoate & $1.29 \pm 0.06^{a}$ & $0.00 \pm 0.00^{\circ}$ & $0.00 \pm 0.00^{c}$ & $0.00 \pm 0.00^{c}$ & $0.00 \pm 0.00^{c}$ & 0.13 & $>1$ & Sweet, cheese \\
\hline Isoamyl hexanoate & $1.35 \pm 0.10^{\mathrm{a}}$ & $0.00 \pm 0.00^{\circ}$ & $0.00 \pm 0.00^{c}$ & $0.00 \pm 0.00^{c}$ & $0.00 \pm 0.00^{c}$ & & & \\
\hline Carbonyl compounds & $2.89 \pm 0.06^{b}$ & $4.64 \pm 0.16^{\mathrm{a}}$ & $2.48 \pm 0.46^{b}$ & $1.90 \pm 0.21^{c}$ & $2.79 \pm 0.51^{b}$ & & & \\
\hline 1-Non-anal & $0.17 \pm 0.01^{b}$ & $0.00 \pm 0.00^{d}$ & $0.27 \pm 0.03^{a}$ & $0.00 \pm 0.00^{d}$ & $0.00 \pm 0.00^{d}$ & 0.015 & $>1$ & Waxy \\
\hline 1-Decanal & $0.32 \pm 0.03^{b}$ & $0.23 \pm 0.05^{\mathrm{c}}$ & $0.00 \pm 0.00^{e}$ & $0.00 \pm 0.00^{e}$ & $0.62 \pm 0.01^{\mathrm{a}}$ & 0.001 & $>1$ & Sweet \\
\hline 2,3-Butanedione & $0.94 \pm 0.02^{d}$ & $3.16 \pm 0.05^{a}$ & $1.52 \pm 0.07^{b}$ & $1.09 \pm 0.06^{c}$ & $1.04 \pm 0.05^{\mathrm{C}}$ & 0.1 & $>1$ & Butter, cheese \\
\hline 2,3-Pentanedione & $1.46 \pm 0.01^{\mathrm{a}}$ & $1.26 \pm 0.07^{\mathrm{ab}}$ & $0.70 \pm 0.36^{c}$ & $0.81 \pm 0.27^{b c}$ & $1.14 \pm 0.47^{\mathrm{abc}}$ & $<0.1$ & $>1$ & Butter, cheese \\
\hline
\end{tabular}

Data show average of triplicates $\pm S D$. Different letters indicated differences among wines determined by Duncan test at the $95 \%$ confidence level.

SCW, S. cerevisiae fermentation; SLY-4W, I. terricola SLY-4 fermentation; F2-24w, P. kudriavzevii F2-24 fermentation; F2-16w, P. kudriavzevii F2-16 fermentation; HX-13w, M. pulcherrima HX-13 fermentation. Bold values represent the total content of a class of substances. 


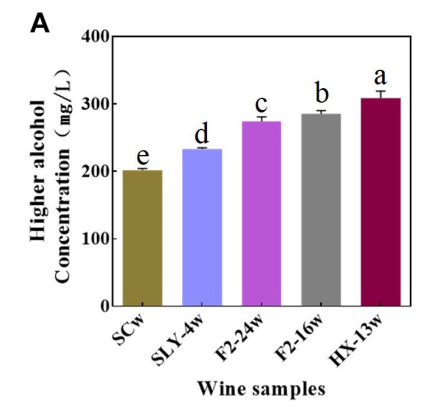

E

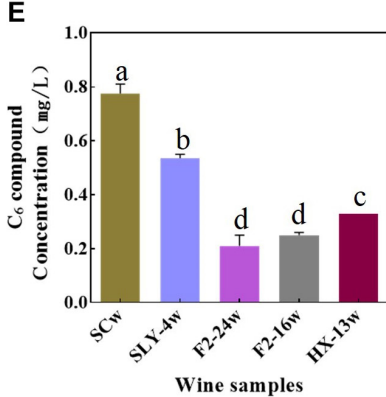

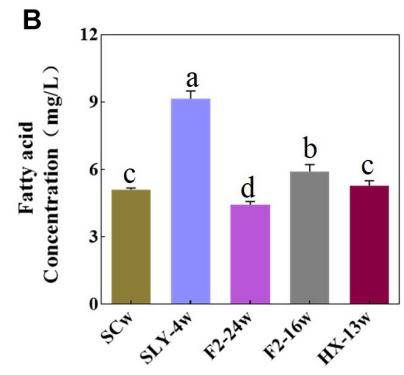

Wine samples

$\mathbf{F}$

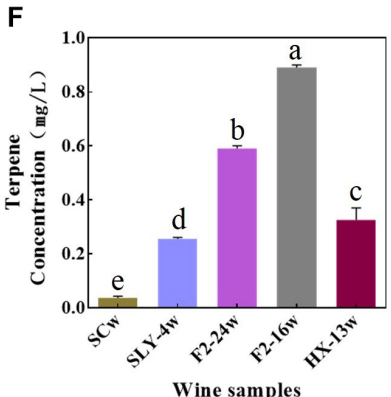

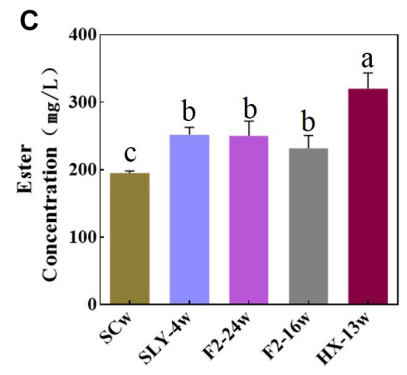

Wine samples

$\mathbf{G}$

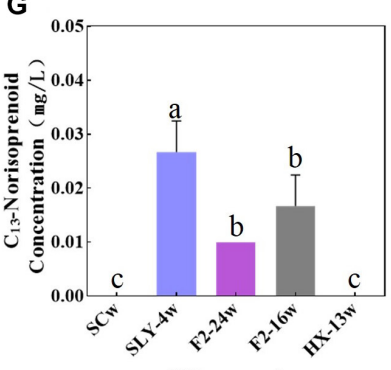

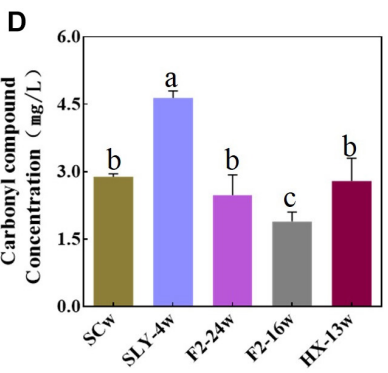

Wine samples

H

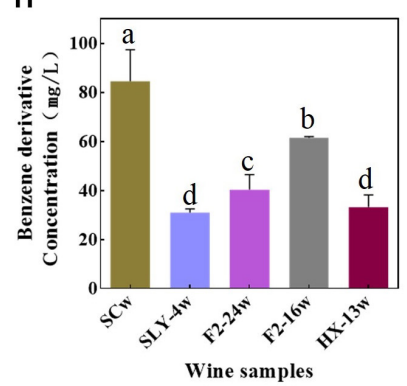

FIGURE 2 | Concentration of aroma compounds in wines fermented by different yeast strains. (A) Higher alcohols. (B) Fatty acids. (C) Esters. (D) Carbonyl compounds. (E) $\mathrm{C}_{6}$ compounds. (F) Terpenes. (G) $\mathrm{C}_{13}$-Norisoprenoids. (H) Benzene derivatives; SCw, S. cerevisiae fermentation; SLY-4w, I. terricola SLY-4 fermentation; F2-24w, P. kudriavzevii F2-24 fermentation; F2-16w, P. kudriavzevii F2-16 fermentation; HX-13w, M. pulcherrima HX-13 fermentation.

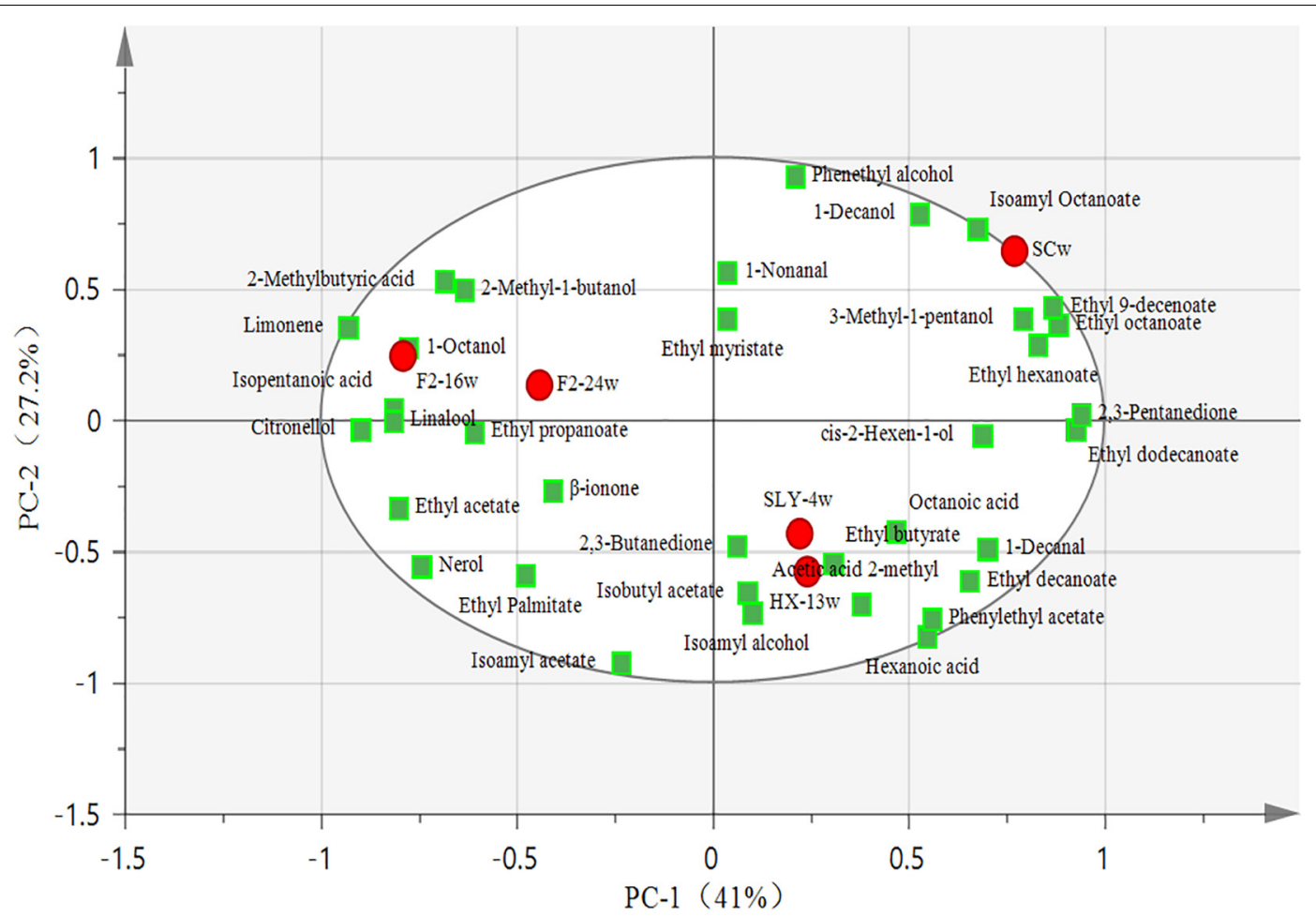

FIGURE 3 | PCA plot of volatile compounds from wines fermented by different yeast strains. Wine samples; $\mathbf{A r o m a}$ compounds; SCW, S. cerevisiae fermentation; SLY-4w, I. terricola SLY-4 fermentation; F2-24w, P. kudriavzevii F2-24 fermentation; F2-16w, P. kudriavzevii F2-16 fermentation; HX-13w, M. pulcherrima $\mathrm{HX}-13$ fermentation. 


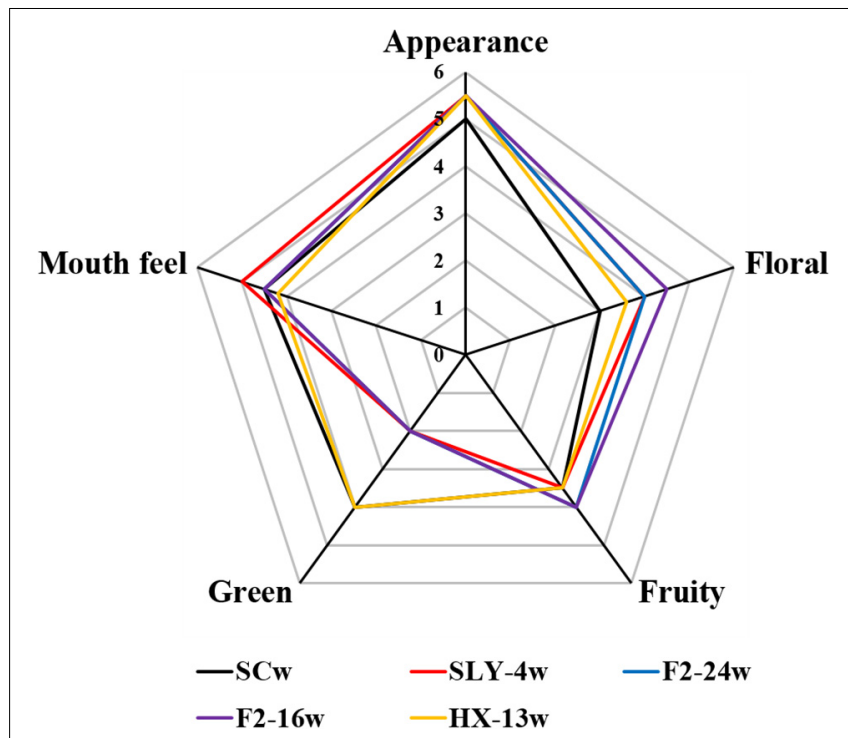

FIGURE 4 | Aroma compounds profiles of wines fermented by different yeast strains with $\beta$-glucosidase activity. SCW, S. cerevisiae fermentation; SLY-4w, 1. terricola SLY-4 fermentation; F2-24w, P. kudriavzevii F2-24 fermentation; F2-16w, P. kudriavzevii F2-16 fermentation; HX-13w, M. pulcherrima HX-13 fermentation.

isoamyl octanoate, 3-methyl-1-pentanol, ethyl 9-decenoate, ethyl octanoate, and ethyl hexanoate (Figure 3).

\section{Sensory Evaluation of Wines Fermented by Different Yeast Strains With $\beta$-Glucosidase Activity}

Compared with that of the $S$. cerevisiae single fermentation, all the non-Saccharomyces yeast fermentations had no significant difference in appearance, but they had a stronger fruity and floral flavor and a weaker green flavor with exception of M. pulcherrima HX-13 fermentation. I. terricola SLY-4 fermentation had the best taste, followed by the fermentations of $S$. cerevisiae, P. kudriavzevii F2-24, P. kudriavzevii F2-16, and M. pulcherrima HX-13. The order of total acceptance for the wines from high to low was fermentation of I. terricola SLY-4, P. kudriavzevii F2-24, P. kudriavzevii F2-16, M. pulcherrima HX-13, and S. cerevisiae (Figure 4).

\section{DISCUSSION}

Compared with those of the $S$. cerevisiae single fermentation, the four non-Saccharomyces yeast strains had higher biomasses and longer fermentation periods which were also reported by Andorrà et al. (2010) and Belda et al. (2015). Those results mean that the four non-Saccharomyces yeast strains consumed sugar more slowly than $S$. cerevisiae did, but they could consume it completely.

Compared with $S$. cerevisiae, the four non-Saccharomyces yeast strains had no negative effects on the chemical characteristics of wines. Specifically, they produced lower contents of $\mathrm{C}_{6}$ compounds and benzene derivative and higher contents of terpene, $\beta$-ionone, higher alcohol, and ester compounds, these phenomena were also reported by Nyanga et al. (2013); Del Mónaco et al. (2014), Lu et al. (2017), and Sun et al. (2017). Low concentrations of $\mathrm{C}_{6}$ compounds would reduce the green flavor of wines (Mendez-Costabel et al., 2014; Vilanova et al., 2016), while high contents of terpene, isoprenoid, benzene derivative, ester, and higher alcohol would enhance the fruity and floral flavor of wines (Pretorius and Lambrechts, 2000; Swiegers and Pretorius, 2005; Noguerol-Pato et al., 2012; Sun et al., 2017). The sensory evaluation results of wines indeed indicated that the green flavor of wines was reduced by the four non-Saccharomyces yeast strains with exception of $M$. pulcherrima HX-13, and their fruity and floral flavors were enhanced. More importantly, the first report about $M$. pulcherrima could produce high content of varietal aroma compounds in wine. The volatile compound profiles of the four non-Sacharomyces yeast fermentations were significantly different from those of $S$. cerevisiae fermentation. Moreover, the volatile compound profiles of $P$. kudriavzevii F2-16 and $P$. kudriavzevii F2-24 fermentations were remarkably different from those of I. terricola SLY-4 and M. pulcherrima HX-13 fermentations. Different volatile compounds profiles would take different flavor characteristics on wines (Lu et al., 2017; Siebert et al., 2018), which was consistent with sensory evaluation results of wines.

\section{CONCLUSION}

Compared with those of $S$. cerevisiae single fermentation, the four non-Saccharomyces yeast strains could grow and consume sugar completely and had no significantly negative effect on chemical characteristics of wines. All the four nonSaccharomyces yeast strains could improve the flavor and quality of wines. Moreover, different yeast strains produced different aroma compounds profiles and take on different aroma characteristics of wines. The four non-Saccharomyces yeast strain fermentations received higher acceptance of sensory evaluation than $S$. cerevisiae did, and $I$. terricola SLY-4 fermentation got the highest sensory evaluation score, followed by $P$. kudriavzevii F2-24, P. kudriavzevii F2-16, and M. pulcherrima HX-13 fermentation from high to low. However, pure non-Saccharomyces yeast fermentation had disadvantages with long fermentation periods and lower content of benzene derivative and fatty acid ethyl ester compounds. To overcome the disadvantages of pure non-Saccharomyces yeast fermentation, co-fermentation of several non-Saccharomyces yeast strains with different aroma compound profiles, or pure $S$. cerevisiae fermentation of must with addition of complex $\beta$-glucosidase (crude or purified) from different non-Saccharomyces yeast strains, might be used to further improve the kind of aroma compounds and the flavor complexity of wine and shorten the fermentation period of wine. The research results will provide non-Saccharomyces yeast strains to improve the flavor and quality of wines, and a reference for the selection of other non-Saccharomyces yeasts strains with better oenological characteristics. 


\section{DATA AVAILABILITY STATEMENT}

The original contributions presented in the study are included in the article/supplementary material, further inquiries can be directed to the corresponding author.

\section{AUTHOR CONTRIBUTIONS}

$\mathrm{XZ}$ and $\mathrm{YZ}$ contributed to the experimental design. $\mathrm{YZ}, \mathrm{WZ}$, and TQ completed the experiments, performed statistical analysis, and wrote the manuscript. TQ and JL contributed to the

\section{REFERENCES}

Andorrà, I., Berradre, M., Rozes, N., Mas, A., Guillamon, J. M., and EsteveZarzoso, B. (2010). Effect of pure and mixed cultures of the main wine yeast species on grape must fermentations. Eur. Food Res. Technol. 231, 215-224. doi: 10.1007/s00217-010-1272-0

Baffi, M. A., Tobal, T., Henrique, J., Lago, G., Leite, R. S. R., Boscolo, M., et al. (2011). A novel $\beta$-glucosidase from. J. Food Sci. 76, 997-1002. doi: 10.1111/j. 1750-3841.2011.02293.x

Belda, I., Navascués, E., Marquina, D., Santos, A., Calderon, F., and Benito, S. (2015). Dynamic analysis of physiological properties of Torulaspora delbrueckii in wine fermentations and its incidence on wine quality. Appl. Microbiol. Biotechnol. 99, 1911-1922. doi: 10.1007/s00253-0146197-2

Binati, R. L., Junior, W. J. F. L., Luzzini, G., Slaghenaufi, D., Ugliano, M., and Torriani, S. (2020). Contribution of non-Saccharomyces yeasts to wine volatile and sensory diversity: a study on Lachancea thermotolerans, Metschnikowia spp. and Starmerella bacillaris strains isolated in Italy - sciencedirect. Int. J. Food Microbiol. 318:108470. doi: 10.1016/j.ijfoodmicro.2019. 108470

Boscaino, F., Ionata, E., Cara, F. L., Guerriero, S., Marcolongo, L., and Sorrentino, A. (2019). Impact of Saccharomyces cerevisiae and Metschnikowia fructicola autochthonous mixed starter on Aglianico wine volatile compounds. J. Food Sci. Technol. 56, 4982-4991. doi: 10.1007/s13197-019-03970-9

Cabrita, M. J., Freitas, A. M. C., Laureano, O., and Stefano, R. D. (2010). Glycosidic aroma compounds of some Portuguese grape cultivars. J. Sci. Food Agric. 86, 922-931. doi: 10.1002/jsfa.2439

Canonico, L., Solomon, M., Comitini, F., Ciani, M., and Varela, C. (2019). Volatile profile of reduced alcohol wines fermented with selected non-Saccharomyces yeasts under different aeration conditions. Food Microbiol. 84, 103247.1103247.8. doi: 10.1016/j.fm.2019.103247

Del Mónaco, S. M., Barda, N. B., Rubio, N. C., and Caballero, A. C. (2014). Selection and characterization of a Patagonian Pichia kudriavzevii for wine deacidification. J. Appl. Microbiol. 117, 451-464. doi: 10.1111/jam.12547

García-Carpintero, E. G., Sánchez-Palomo, E., and González-Viñas, M. A. (2011). Aroma characterization of red wines from cv. Bobal grape variety grown in La Mancha region. Food Res. Int. 44, 61-70. doi: 10.1016/j.foodres.2010. 11.013

López, M. C., Mateo, J. J., and Maicas, S. (2015). Screening of $\beta$-glucosidase and $\beta$-xylosidase activities in four non-Saccharomyces yeast isolates. J. Food Sci. 80, C1696-C1704. doi: 10.1111/1750-3841.12954

López, S., Mateo, J. J., and Maicas, S. M. (2016). Characterisation of Hanseniaspora isolates with potential aroma-enhancing properties in muscat wines. S. Afr. J. Enol. Viticult. 35, 292-303. doi: 10.21548/35-21018

Lu, Y., Voon, M. K. W., Chua, J. Y., Huang, D., Lee, P. R., and Liu, S. Q. (2017). The effects of co- and sequential inoculation of Torulaspora delbrueckii and Pichia kluyveri on chemical compositions of durian wine. Appl. Microbiol. Biotechnol. 101, 7853-7863. doi: 10.1007/s00253-017-8527-7

Ma, D., Xia, Y., Wang, Q., Zhang, Y., and Tao, Y. (2017). Performance of selected $P$. fermentans and its excellular enzyme in co-inoculation with $S$. cerevisiae for experiment verification. JL, YZ, and TQ contributed to the revision of the manuscript. All authors contributed to the article and approved the submitted version.

\section{FUNDING}

This research was supported by the National Natural Science Foundation Project of China (31871768) and the Major Science and Technology Program of Ningxia Hui Autonomous Region (2016BZ0601/02/03).

wine aroma enhancement. LWT Food Sci. Technol. 86, 361-370. doi: 10.1016/j. lwt.2017.08.018

Mendez-Costabel, M. P., Wilkinson, K. L., Bastian, S. E. P., Jordans, C., Mccarthy, M., Ford, C. M., et al. (2014). Effect of increased irrigation and additional nitrogen fertilisation on the concentration of green aroma compounds in Vitis vinifera L. Merlot fruit and wine. Aust. J. Grape Wine R. 20, 80-90. doi: 10.1111/ ajgw. 12062

Noguerol-Pato, R., González-Álvarez, M., González-Barreiro, C., Cancho-Grande, B., and Simal-Gándara, J. (2012). Aroma profile of Garnacha Tintorera-based sweet wines by chromatographic and sensorial analyses. Food Chem. 134, 2313-2325. doi: 10.1016/j.foodchem.2012.03.105

Nyanga, L. K., Nout, M. J. R., Smid, E. J., Teun, B., and Zwietering, M. H. (2013). Fermentation characteristics of yeasts isolated from traditionally fermented masau (Ziziphus mauritiana) fruits. Int. J. Food Microbiol. 166, 426-432. doi: 10.1016/j.ijfoodmicro.2013.08.003

OIV-MA-AS313-01: R2015. (2015). Compendium of International Methods of Wine and Must Analysis. Chemical Analysis: Acids, Total Acidity (Oeno 551/2015). Paris: Organisation Internationale de la Vigne et du Vin.

OIV-MA-AS312-01A: R2016. (2016). Compendium of International Methods of Wine and Must Analysis. Chemical Analysis: Alcohols, Alcoholic Strength by Volume (Resolution Oeno 566/2016). Paris: Organisation Internationale de la Vigne et du Vin.

OIV-MA-AS311-02: R2009. (2009). Compendium of International Methods of Wine and Must Analysis. Chemical Analysis: SUGARS, Glucose and Fructose. Paris: Organisation Internationale de la Vigne et du Vin.

OIV-MA-AS313-02: R2015. (2015). Compendium of International Methods of Wine and Must Analysis. Chemical Analysis: Acids, Volatile Acidity. Paris: Organisation Internationale de la Vigne et du Vin.

Ovalle, S. D., Brena, B., Fariña, L., and Gonzálezpombo, P. (2016). Novel $\beta$ glucosidase from Issatchenkia orientalis: characterization and assessment for hydrolysis of muscat wine glycosides. Global J. Biochem. Biotechnol. 4, 174-183. doi: 10.1016/j.lwt.2017.09.026

Parker, M., Capone, D. L., Francis, I. L., and Herderich, M. J. (2017). Aroma precursors in grapes and wine: flavor release during wine production and consumption. J. Agric. Food Chem. 66, 2281-2286. doi: 10.1021/acs.jafc. 6b05255

Pires, E. J., Teixeira, J. A., Brányik, T., and Vicente, A. A. (2014). Yeast: the soul of beer's aroma-a review of flavour-active esters and higher alcohols produced by the brewing yeast. Appl. Microbiol. Biotechnol. 98, 1937-1949. doi: 10.1007/s00253-013-5470-0

Plessis, H. D., Toit, M. D., Nieuwoudt, H. H., Rijst, M. V. D., and Jolly, N. (2019). Modulation of wine flavor using Hanseniaspora uvarum in combination with different Saccharomyces cerevisiae, lactic acid bacteria strains and malolactic fermentation strategies. Fermentation 5:64. doi: 10.3390/fermentation50 30064

Pretorius, I. S., and Lambrechts, M. G. (2000). Yeast and its importance to wine aroma: a review. South Afr. J. Enol. Viticult. 21, 97-129. doi: 10.21548/21-13560

Sabel, A., Martens, S., Petri, A., König, H., and Claus, H. (2014). Wickerhamomyces anomalus as1: a new strain with potential to improve wine aroma. Ann. Microbiol. 64, 483-491. doi: 10.1007/s13213-013-0678-x 
Siebert, T. E., Barker, A., Pearson, W., Barter, S. R., Ma, D. B. L., Darriet, P., et al. (2018). Volatile compounds related to 'Stone Fruit' aroma attributes in viognier and chardonnay wines. J. Agric. Food Chem. 66, 2838-2850. doi: 10.1021/acs. jafc. $7 \mathrm{~b} 05343$

Suárez, R., Suárez-Lepe, J. A., Morata, A., and Calderón, F. (2007). The production of ethylphenols in wine by yeasts of the genera Brettanomyces and Dekkera: a review. Food Chem. 102, 10-21. doi: 10.1016/j.foodchem.2006.03.030

Sun, W. X., Hu, K., Zhang, J. X., Zhu, X. L., and Tao, Y. S. (2017). Aroma modulation of Cabernet Gernischt dry red wine by optimal enzyme treatment strategy in winemaking. Food Chem. 245:1248. doi: 10.1016/j.foodchem.2017. 11.106

Swiegers, J. H., and Pretorius, I. S. (2005). Yeast modulation of wine flavor. $A d v$. Appl. Microbiol. 57, 131-175. doi: 10.1016/S0065-2164(05)57005-9

Vilanova, M., Genisheva, Z., Graña, M., and Oliveira, J. M. (2016). Determination of odorants in varietal wines from international grape cultivars (Vitis vinifera) grown in NW Spain. S. Afr. J. Enol. Vitic. 34, 6502-6506. doi: 10.1073/pnas.87. 17.6502

Wang, J., Hu, L. L., Zhang, J. X., Chen, F. S., and Zhang, X. Y. (2018). Screening of wild yeast with high yield $\beta$-glycosidase and difference analysis of the enzyme production ability. Chn. Brw. 037, 50-53. doi: 10.11882/j.issn.0254-5071.2018. 02.011
Zhang, W., Zhuo, X., Hu, L., and Zhang, X. (2020). Effects of crude $\beta$-glucosidases from Issatchenkia terricola, Pichia kudriavzevii, Metschnikowia pulcherrima on the flavor complexity and characteristics of wines. Microorganisms 8:953. doi: 10.3390/microorganisms 8060953

Conflict of Interest: The authors declare that the research was conducted in the absence of any commercial or financial relationships that could be construed as a potential conflict of interest.

Publisher's Note: All claims expressed in this article are solely those of the authors and do not necessarily represent those of their affiliated organizations, or those of the publisher, the editors and the reviewers. Any product that may be evaluated in this article, or claim that may be made by its manufacturer, is not guaranteed or endorsed by the publisher.

Copyright $\odot 2021$ Qin, Liao, Zheng, Zhang and Zhang. This is an open-access article distributed under the terms of the Creative Commons Attribution License (CC BY). The use, distribution or reproduction in other forums is permitted, provided the original author(s) and the copyright owner(s) are credited and that the original publication in this journal is cited, in accordance with accepted academic practice. No use, distribution or reproduction is permitted which does not comply with these terms. 